\title{
ON SEMI-INVERSE SOLUTIONS FOR THE TIME-DEPENDENT FLOWS OF A SECOND-GRADE FLUID
}

\author{
MUHAMMAD R. MOHYUDDIN, S. ASGHAR, T. HAYAT, AND A. M. SIDDIQUI
}

Received 7 June 2005; Revised 16 December 2005; Accepted 15 January 2006

This paper deals with analytical solutions for the time-dependent equations arising in a second-grade fluid. The solutions have been developed by assuming certain forms of the stream function. Expressions for velocity components are obtained for flows in plane polar, axisymmetric cylindrical, and axisymmetric spherical polar coordinates. The obtained solutions are compared with existing results.

Copyright (c) 2006 Muhammad R. Mohyuddin et al. This is an open access article distributed under the Creative Commons Attribution License, which permits unrestricted use, distribution, and reproduction in any medium, provided the original work is properly cited.

\section{Introduction}

Under several assumptions, the Navier-Stokes equations can be linearized, and closedform solutions are available. It is known that in general, the governing equations of nonNewtonian fluids are nonlinear and much more complicated in comparison to Newtonain fluids. To obtain analytical solutions of such equations is not easy. In order to explain several nonstandard features, such as normal-stress effects, rod climbing, shear thinning, and shear thickening, Rivlin-Ericksen fluids [18] of differential type were introduced. The second-grade fluids form a subclass of the differential-type fluid. The Cauchy stress $\mathrm{T}$ in a second-grade fluid is $[1,11,12,15,19]$

$$
\mathbf{T}=-p \mathbf{I}+\mu \mathbf{A}_{1}+\alpha_{1} \mathbf{A}_{2}+\alpha_{2} \mathbf{A}_{1}^{2}
$$

where

$$
\begin{gathered}
\mathbf{A}_{1}=(\operatorname{grad} \mathbf{V})+(\operatorname{grad} \mathbf{V})^{*}, \\
\mathbf{A}_{2}=\frac{d \mathbf{A}_{1}}{d t}+\mathbf{A}_{1}(\operatorname{grad} \mathbf{V})+(\operatorname{grad} \mathbf{V})^{*} \mathbf{A}_{1}
\end{gathered}
$$

in which $\mathbf{V}$ is the velocity vector, $(*)$ is the matrix transpose, $p$ is the indeterminate pressure constrained by the incompressibility, $d / d t$ is the material derivative, $\mu, \alpha_{1}$, and 
$\alpha_{2}$ are material constants. They denote, respectively, the viscosity, elasticity, and crossviscosity. These material constants can be determined from viscometric flows for any real fluid. Second-grade fluids are dilute polymers and the differential-type models can be used to describe the response of dilute polymers. A detailed account of the characteristics of second-order fluids is well documented by Dunn and Rajagopal [4]. Fosdick and Rajagopal [5] have studied that the relation (1.1) is compatible with thermodynamics (the Clausius-Duhem inequality and the assumption that the Helmholtz free energy is a minimum in equilibrium when the fluid is at rest) if the following restrictions are imposed on the material constants:

$$
\mu \geq 0, \quad \alpha_{1} \geq 0, \quad \alpha_{1}+\alpha_{2}=0 .
$$

By assuming a certain form of the stream function, solutions for second-grade fluids were obtained by Mohyuddin et al. [10], Kaloni and Huschilt [7], Siddiqui et al. [2, 19, 20], Hayat et al. [6], and Labropulu [8].

The main purpose of the present communication is twofold. Firstly, to present the equations for the unsteady plane and axisymmetric flows of a second-grade fluids in polar, cylindrical, and spherical coordinates. Secondly, to obtain some analytical solutions of the governing equations in each case. We mention here that no boundary value problem is considered. The governing equations of the second-grade fluids are highly nonlinear. Moreover, these equations are of higher order than the Navier-Stokes equations. Thus, in general, to solve a well-posed problem for such a fluid, one requires additional initial and/or boundary conditions. For a detailed discussion about this issue and for some interesting examples, we refer the readers to the works by Rajagopal and Gupta [16], Rajagopal and Kaloni [17], and Rajagopal [13, 14]. The semi-inverse approach is used to deduce solutions of the governing time-dependent equations.

The outline of the paper is as follows. In Section 2, the basic laws are given. Section 3 contains the modelling for unsteady flows for three cases. In Section 4, analytic solutions are calculated for each case. The analytical solutions obtained are new and comparisons are made with the solutions available in the literature.

\section{Basic equations}

The basic laws governing the motion of an incompressible, homogeneous, second-grade fluid are

$$
\begin{gathered}
\operatorname{div} \mathbf{V}=0, \\
-\operatorname{grad} p+\operatorname{div}\left(\mu \mathbf{A}_{1}+\alpha_{1} \mathbf{A}_{2}+\alpha_{2} \mathbf{A}_{1}^{2}\right)+\rho \mathbf{b}=\rho \frac{d \mathbf{V}}{d t},
\end{gathered}
$$

where div is the divergence and grad is the gradient operator. Substituting (1.2) in (2.2), we obtain

$$
\begin{array}{r}
\operatorname{grad}\left[\frac{1}{2} \rho|\mathbf{V}|^{2}+p-\alpha_{1}\left(\mathbf{V} \cdot \nabla^{2} \mathbf{V}+\frac{1}{4}\left|\mathbf{A}_{1}\right|^{2}\right)\right]+\rho\left[\mathbf{V}_{t}-\mathbf{V} \times(\nabla \times \mathbf{V})\right] \\
=\mu \nabla^{2} \mathbf{V}+\alpha_{1}\left[\nabla^{2} \mathbf{V}_{t}+\nabla^{2}(\nabla \times \mathbf{V}) \times \mathbf{V}\right]+\left(\alpha_{1}+\alpha_{2}\right) \operatorname{div} \mathbf{A}_{1}^{2}+\rho \mathbf{b},
\end{array}
$$


where $t$ in the subscript denotes the partial derivative with respect to time and $\nabla^{2}$ is the Laplacian operator.

\section{Compatibility equations}

In this section, we give the compatibility equations in plane polar, axisymmetric cylindrical, and axisymmetric spherical polar coordinates. The equations are constructed by eliminating the pressure field from the resulting equations in component form of (2.3) along with the continuity equation (2.1).

3.1. Unsteady plane flows in polar coordinates. Here, the velocity field is of the following form:

$$
\mathbf{V}=[u(r, \theta, t), v(r, \theta, t), 0]
$$

Making use of (3.1) into (2.1) and (2.3), we obtain in the absence of body forces

$$
\begin{gathered}
\frac{\partial u}{\partial r}+\frac{u}{r}+\frac{1}{r} \frac{\partial v}{\partial \theta}=0 \\
\frac{\partial \hat{p}}{\partial r}+\rho\left[\frac{\partial u}{\partial t}-v \omega\right]=-\frac{1}{r}\left(\mu+\alpha_{1} \frac{\partial}{\partial t}\right) \frac{\partial \omega}{\partial \theta}-\alpha_{1} v \nabla^{2} \omega \\
\frac{1}{r} \frac{\partial \hat{p}}{\partial \theta}+\rho\left[\frac{\partial v}{\partial t}+u \omega\right]=\left(\mu+\alpha_{1} \frac{\partial}{\partial t}\right) \frac{\partial \omega}{\partial r}+\alpha_{1} u \nabla^{2} \omega
\end{gathered}
$$

in which $\hat{p}$ is the modified pressure and $\omega$ is the vorticity vector. The expressions for $\hat{p}$ and $\omega$ are

$$
\begin{gathered}
\hat{p}=p+\frac{1}{2} \rho\left(u^{2}+v^{2}\right)+\alpha_{1}\left(\frac{u}{r} \frac{\partial \omega}{\partial \theta}-v \frac{\partial \omega}{\partial r}\right)-\frac{\left(3 \alpha_{1}+2 \alpha_{2}\right)}{4}\left|\mathbf{A}_{1}^{2}\right| \\
\left|\mathbf{A}_{1}^{2}\right|=4\left(\frac{\partial u}{\partial r}\right)^{2}+4\left(\frac{1}{r} \frac{\partial v}{\partial \theta}+\frac{u}{r}\right)^{2}+2\left(\frac{\partial v}{\partial r}-\frac{v}{r}+\frac{1}{r} \frac{\partial u}{\partial \theta}\right)^{2} \\
\omega=\frac{\partial v}{\partial r}+\frac{v}{r}-\frac{1}{r} \frac{\partial u}{\partial \theta} \\
\nabla^{2}=\frac{\partial^{2}}{\partial r^{2}}+\frac{1}{r} \frac{\partial}{\partial r}+\frac{1}{r^{2}} \frac{\partial^{2}}{\partial \theta^{2}} .
\end{gathered}
$$

The existence of continuity equation (2.1) allows us to define a stream function $\psi(r, \theta, t)$ as

$$
u=\frac{1}{r} \frac{\partial \psi}{\partial \theta}, \quad v=-\frac{\partial \psi}{\partial r}
$$


4 Unsteady semi-inverse solutions

which satisfies (3.2) identically and (3.3) and (3.4) become

$$
\begin{aligned}
& \frac{\partial \hat{p}}{\partial r}+\rho\left[\frac{1}{r} \frac{\partial^{2} \psi}{\partial t \partial \theta}-\frac{\partial \psi}{\partial r} \nabla^{2} \psi\right]=\frac{1}{r}\left(\mu+\alpha_{1} \frac{\partial}{\partial t}\right) \frac{\partial\left(\nabla^{2} \psi\right)}{\partial \theta}-\alpha_{1} \frac{\partial \psi}{\partial r} \nabla^{4} \psi, \\
& \frac{\partial \hat{p}}{\partial \theta}-\rho\left[r \frac{\partial^{2} \psi}{\partial t \partial r}+\frac{\partial \psi}{\partial \theta} \nabla^{2} \psi\right]=-r\left(\mu+\alpha_{1} \frac{\partial}{\partial t}\right) \frac{\partial\left(\nabla^{2} \psi\right)}{\partial r}-\alpha_{1} \frac{\partial \psi}{\partial \theta} \nabla^{4} \psi,
\end{aligned}
$$

where $\omega=-\nabla^{2} \psi$. Differentiating (3.7) with respect to $\theta$ and (3.8) with respect to $r$ and using the integrability condition $\hat{p}_{r \theta}=\hat{p}_{\theta r}$, we obtain

$$
\rho\left[r \frac{\partial}{\partial t} \nabla^{2} \psi-\left\{\psi, \nabla^{2} \psi\right\}\right]=r\left(\mu+\alpha_{1} \frac{\partial}{\partial t}\right) \nabla^{4} \psi-\alpha_{1}\left\{\psi, \nabla^{4} \psi\right\}
$$

in which

$$
\left\{\psi, \nabla^{2} \psi\right\}=\frac{\partial \psi}{\partial r} \frac{\partial\left(\nabla^{2} \psi\right)}{\partial \theta}-\frac{\partial \psi}{\partial \theta} \frac{\partial\left(\nabla^{2} \psi\right)}{\partial r} .
$$

It is to be noted that the material parameter $\alpha_{2}$ does not contribute to the compatibility equation (3.9). However, it does appear in the pressure distribution given in (3.5a).

3.2. Unsteady axisymmetric flows in cylindrical coordinates. The velocity field for this case is

$$
\mathbf{V}=[u(r, z, t), 0, w(r, z, t)]
$$

The continuity and momentum equations are

$$
\begin{aligned}
& \frac{\partial u}{\partial r}+\frac{u}{r}+\frac{\partial w}{\partial z}=0 \\
& \frac{\partial \tilde{p}}{\partial r}+\rho\left[\frac{\partial u}{\partial t}-w \Omega\right] \\
& \quad=-\left(\mu+\alpha_{1} \frac{\partial}{\partial t}\right) \frac{\partial \Omega}{\partial z}-\alpha_{1} w\left(\nabla^{2} \Omega-\frac{\Omega}{r^{2}}\right)+\frac{1}{r}\left(\alpha_{1}+\alpha_{2}\right)\left[2 \frac{\partial}{\partial z}(u \Omega)+\Omega^{2}\right], \\
& \frac{\partial \tilde{p}}{\partial z}+\rho\left[\frac{\partial w}{\partial t}+u \Omega\right]=\left(\mu+\alpha_{1} \frac{\partial}{\partial t}\right)\left(\frac{\partial \Omega}{\partial r}+\frac{\Omega}{r}\right)+\alpha_{1} u\left(\nabla^{2} \Omega-\frac{\Omega}{r^{2}}\right)-\frac{2}{r}\left(\alpha_{1}+\alpha_{2}\right) \frac{\partial}{\partial r}(u \Omega),
\end{aligned}
$$

where

$$
\begin{gathered}
\tilde{p}=p+\frac{1}{2} \rho\left(u^{2}+w^{2}\right)+\alpha_{1}\left[u\left(\nabla^{2} u-\frac{u}{r^{2}}\right)+w \nabla^{2} w\right]-\frac{\left(3 \alpha_{1}+2 \alpha_{2}\right)}{4}\left|\mathbf{A}_{1}^{2}\right| \\
\left|\mathbf{A}_{1}^{2}\right|=4\left(\frac{\partial u}{\partial r}\right)^{2}+4\left(\frac{\partial w}{\partial z}\right)^{2}+4\left(\frac{u}{r}\right)^{2}+2\left(\frac{\partial u}{\partial z}+\frac{\partial w}{\partial r}\right)^{2} \\
\Omega=\frac{\partial w}{\partial r}-\frac{\partial u}{\partial z}, \quad \nabla^{2}=\frac{\partial^{2}}{\partial r^{2}}+\frac{1}{r} \frac{\partial}{\partial r}+\frac{\partial^{2}}{\partial z^{2}}
\end{gathered}
$$


Introducing the stream function $\widetilde{\psi}(r, z, t)$ through

$$
u=\frac{1}{r} \frac{\partial \tilde{\psi}}{\partial z}, \quad w=-\frac{1}{r} \frac{\partial \tilde{\psi}}{\partial r}
$$

the continuity equation (3.12) is satisfied identically and (3.13) give

$$
\begin{aligned}
\frac{\partial \tilde{p}}{\partial r}+ & \rho\left[\frac{1}{r} \frac{\partial^{2} \tilde{\psi}}{\partial t \partial z}-\frac{\partial \tilde{\psi}}{\partial r} \frac{E^{2} \tilde{\psi}}{r^{2}}\right] \\
& =\frac{1}{r}\left(\mu+\alpha_{1} \frac{\partial}{\partial t}\right) \frac{\partial\left(E^{2} \tilde{\psi}\right)}{\partial z}-\alpha_{1} \frac{\partial \tilde{\psi}}{\partial r} \frac{E^{4} \tilde{\psi}}{r^{2}}-\frac{1}{r}\left(\alpha_{1}+\alpha_{2}\right)\left[2 \frac{\partial}{\partial z}\left(\frac{\partial \tilde{\psi}}{\partial z} \frac{E^{2} \tilde{\psi}}{r^{2}}\right)-\left(\frac{E^{2} \tilde{\psi}}{r}\right)^{2}\right], \\
\frac{\partial \tilde{p}}{\partial z}-\rho\left[\frac{1}{r} \frac{\partial^{2} \tilde{\psi}}{\partial t \partial r}+\frac{\partial \tilde{\psi}}{\partial z} \frac{E^{2} \tilde{\psi}}{r^{2}}\right] & -\frac{1}{r}\left(\mu+\alpha_{1} \frac{\partial}{\partial t}\right) \frac{\partial\left(E^{2} \tilde{\psi}\right)}{\partial r}-\alpha_{1} \frac{\partial \tilde{\psi}}{\partial z} \frac{E^{4} \tilde{\psi}}{r^{2}}+\frac{2}{r}\left(\alpha_{1}+\alpha_{2}\right) \frac{\partial}{\partial r}\left(\frac{\partial \tilde{\psi}}{\partial z} \frac{E^{2} \tilde{\psi}}{r^{2}}\right),
\end{aligned}
$$

where

$$
E^{2}=\frac{\partial^{2}}{\partial r^{2}}-\frac{1}{r} \frac{\partial}{\partial r}+\frac{\partial^{2}}{\partial z^{2}}, \quad \Omega=-\frac{1}{r} E^{2} \widetilde{\psi}
$$

Elimination of $\tilde{p}$ from the above equations yields

$$
\begin{aligned}
& \rho\left[\frac{1}{r} \frac{\partial}{\partial t} E^{2} \tilde{\psi}-\left\{\tilde{\psi}, \frac{E^{2} \tilde{\psi}}{r^{2}}\right\}\right] \\
& \quad=\frac{1}{r}\left(\mu+\alpha_{1} \frac{\partial}{\partial t}\right) E^{4} \tilde{\psi}-\alpha_{1}\left\{\tilde{\psi}, \frac{E^{4} \tilde{\psi}}{r^{2}}\right\}-\frac{1}{r}\left(\alpha_{1}+\alpha_{2}\right)\left[2 E^{2}\left(\frac{\partial \tilde{\psi}}{\partial z} \frac{E^{2} \tilde{\psi}}{r^{2}}\right)-\frac{\partial}{\partial z}\left(\frac{E^{2} \tilde{\psi}}{r}\right)^{2}\right],
\end{aligned}
$$

in which

$$
\left\{\tilde{\psi}, \frac{E^{4} \tilde{\psi}}{r^{2}}\right\}=\frac{\partial \tilde{\psi}}{\partial r} \frac{\partial}{\partial z}\left(\frac{E^{4} \tilde{\psi}}{r^{2}}\right)-\frac{\partial \tilde{\psi}}{\partial z} \frac{\partial}{\partial r}\left(\frac{E^{4} \tilde{\psi}}{r^{2}}\right) .
$$

3.3. Unsteady axisymmetric flows in spherical coordinates. On using the velocity field

$$
\mathbf{V}=[u(R, \theta, t), v(R, \theta, t), 0]
$$


into (2.1) and (2.3), we obtain the following equations:

$$
\begin{aligned}
& \frac{\partial u}{\partial R}+\frac{2 u}{R}+\frac{1}{R} \frac{\partial v}{\partial \theta}+\frac{v}{R} \cot \theta=0, \\
& \frac{\partial \bar{p}}{\partial R}+\rho\left[\frac{\partial u}{\partial t}-v \bar{\Omega}\right]=-\left(\mu+\alpha_{1} \frac{\partial}{\partial t}\right) \frac{1}{R \sin \theta} \frac{\partial}{\partial \theta}(\bar{\Omega} \sin \theta)-\alpha_{1} v\left(\nabla^{2} \bar{\Omega}-\frac{\bar{\Omega}}{R^{2} \sin ^{2} \theta}\right) \\
& +\left(\alpha_{1}+\alpha_{2}\right)\left[\frac{2}{R \sin \theta} \frac{\partial}{\partial \theta}\left(\frac{u}{R}+\frac{v}{R} \cot \theta\right) \bar{\Omega} \sin \theta+\frac{\bar{\Omega}^{2}}{R}\right], \\
& \frac{1}{R} \frac{\partial \bar{p}}{\partial \theta}+\rho\left[\frac{\partial v}{\partial t}+u \bar{\Omega}\right]=\left(\mu+\alpha_{1} \frac{\partial}{\partial t}\right)\left(\frac{\partial \bar{\Omega}}{\partial R}+\frac{\bar{\Omega}}{R}\right)+\alpha_{1} u\left(\nabla^{2} \bar{\Omega}-\frac{\bar{\Omega}}{R^{2} \sin ^{2} \theta}\right) \\
& +\frac{\left(\alpha_{1}+\alpha_{2}\right)}{R}\left[-2 \frac{\partial}{\partial R}(u+v \cot \theta) \bar{\Omega}+\bar{\Omega}^{2} \cot \theta\right],
\end{aligned}
$$

where

$$
\begin{gathered}
\bar{p}=p+\frac{1}{2} \rho\left(u^{2}+v^{2}\right)-\alpha_{1}\left[\begin{array}{c}
u\left(\nabla^{2} u-\frac{2 u}{R^{2}}+\frac{2}{R^{2}} \frac{\partial v}{\partial \theta}-\frac{2 v}{R^{2}} \cot \theta\right) \\
+v\left(\nabla^{2} u+\frac{2}{R^{2}} \frac{\partial u}{\partial \theta}-\frac{v}{R^{2} \sin ^{2} \theta}\right)
\end{array}\right]-\frac{\left(3 \alpha_{1}+2 \alpha_{2}\right)}{4}\left|\mathbf{A}_{1}^{2}\right|, \\
\left|\mathbf{A}_{1}^{2}\right|=4\left(\frac{\partial u}{\partial R}\right)^{2}+4\left(\frac{1}{R} \frac{\partial v}{\partial \theta}+\frac{u}{R}\right)^{2}+4\left(\frac{u}{R}+\frac{v}{R} \cot \theta\right)^{2}+2\left(\frac{\partial v}{\partial R}-\frac{v}{R}+\frac{1}{R} \frac{\partial u}{\partial \theta}\right)^{2} \\
\bar{\Omega}=\frac{\partial v}{\partial R}+\frac{v}{R}-\frac{1}{R} \frac{\partial u}{\partial \theta}, \quad \nabla^{2}=\frac{1}{R^{2}} \frac{\partial}{\partial R}\left(R^{2} \frac{\partial}{\partial R}\right)+\frac{1}{R^{2} \sin \theta} \frac{\partial}{\partial \theta}\left(\sin \theta \frac{\partial}{\partial \theta}\right) .
\end{gathered}
$$

Define the stream function in spherical polar coordinates as

$$
u=\frac{1}{R^{2}} \frac{\partial \bar{\psi}}{\partial \sigma}, \quad v=\frac{1}{R \sqrt{1-\sigma^{2}}} \frac{\partial \bar{\psi}}{\partial R}, \quad \sigma=\cos \theta
$$

We see that (3.21) is satisfied identically and (3.22) lead to

$$
\begin{aligned}
\frac{\partial \bar{p}}{\partial R}= & -\rho\left[\frac{1}{R^{2}} \frac{\partial^{2} \bar{\psi}}{\partial t \partial \sigma}-\frac{1}{R^{2}\left(1-\sigma^{2}\right)} \frac{\partial \bar{\psi}}{\partial R} D^{2} \bar{\psi}\right]+\frac{1}{R^{2}}\left(\mu+\alpha_{1} \frac{\partial}{\partial t}\right) D^{2} \bar{\psi}-\frac{\alpha_{1}}{R^{2}\left(1-\sigma^{2}\right)} \frac{\partial \bar{\psi}}{\partial R} D^{4} \bar{\psi} \\
& +\left(\alpha_{1}+\alpha_{2}\right)\left[\begin{array}{c}
\left.-\frac{2}{R} \frac{\partial}{\partial \sigma}\left\{\left(\frac{1}{R^{4}} \frac{\partial \bar{\psi}}{\partial \sigma}+\frac{\sigma}{R^{3}\left(1-\sigma^{2}\right)} \frac{\partial \bar{\psi}}{\partial R}\right) D^{2} \bar{\psi}\right\}\right] \\
+\frac{\left(D^{4} \bar{\psi}\right) 2}{R^{3}\left(1-\sigma^{2}\right)}
\end{array}\right],
\end{aligned}
$$




$$
\begin{aligned}
\frac{\partial \bar{p}}{\partial \sigma}= & -\frac{\rho}{1-\sigma^{2}}\left[\frac{\partial^{2} \bar{\psi}}{\partial t \partial R}+\frac{1}{R^{2}} \frac{\partial \bar{\psi}}{\partial \sigma} D^{2} \bar{\psi}\right] \\
& +\frac{1}{1-\sigma^{2}}\left(\mu+\alpha_{1} \frac{\partial}{\partial t}\right) \frac{\partial\left(D^{2} \bar{\psi}\right)}{\partial R}+\frac{\alpha_{1}}{R^{2}\left(1-\sigma^{2}\right)} \frac{\partial \bar{\psi}}{\partial \sigma} D^{4} \bar{\psi} \\
& -\frac{\left(\alpha_{1}+\alpha_{2}\right)}{\sqrt{1-\sigma^{2}}}\left[\begin{array}{c}
2 \frac{\partial}{\partial R}\left(\frac{1}{R^{3}} \frac{\partial \bar{\psi}}{\partial \sigma}+\frac{\sigma}{R^{2}\left(1-\sigma^{2}\right)} \frac{\partial \bar{\psi}}{\partial R}\right) \frac{D^{2} \bar{\psi}}{\sqrt{1-\sigma^{2}}} \\
-\frac{\sigma\left(D^{2} \bar{\psi}\right)^{2}}{R^{2}\left(1-\sigma^{2}\right)^{3 / 2}}
\end{array}\right],
\end{aligned}
$$

where

$$
D^{2}=\frac{\partial^{2}}{\partial R^{2}}+\frac{1-\sigma^{2}}{R^{2}} \frac{\partial^{2}}{\partial \sigma^{2}} .
$$

Differentiating (3.25) with respect to $\sigma$ and (3.26) with respect to $R$ and using integrability condition $\bar{p}_{R \sigma}=\bar{p}_{\sigma R}$, we obtain

$$
\begin{aligned}
\rho\left[\begin{array}{c}
\frac{\partial}{\partial t}\left(\frac{D^{2} \bar{\psi}}{1-\sigma^{2}}\right) \\
-\left\{\bar{\psi}, \frac{D^{2} \bar{\psi}}{R^{2}\left(1-\sigma^{2}\right)}\right\}
\end{array}\right]=\left(\mu+\alpha_{1} \frac{\partial}{\partial t}\right) \frac{D^{4} \bar{\psi}}{1-\sigma^{2}}-\alpha_{1}\left\{\bar{\psi}, \frac{D^{4} \bar{\psi}}{R^{2}\left(1-\sigma^{2}\right)}\right\} \\
+\frac{2\left(\alpha_{1}+\alpha_{2}\right)}{1-\sigma^{2}}\left[\begin{array}{c}
D^{2}\left(\begin{array}{c}
\frac{\partial \bar{\psi}}{\partial \sigma} \frac{D^{2} \bar{\psi}}{R^{3}} \\
+\frac{\sigma}{R^{2}\left(1-\sigma^{2}\right)} \frac{\partial \bar{\psi}}{\partial R} D^{2} \bar{\psi}
\end{array}\right) \\
-\left(\begin{array}{c}
\frac{D^{2} \bar{\psi}}{R^{3}} \frac{\partial}{\partial \sigma} \\
+\frac{\sigma D^{2} \bar{\psi}}{R^{2}\left(1-\sigma^{2}\right)} \frac{\partial}{\partial R}
\end{array}\right) D^{2} \bar{\psi}
\end{array}\right],
\end{aligned}
$$

in which

$$
\left\{\bar{\psi}, \frac{D^{2} \bar{\psi}}{R^{2}\left(1-\sigma^{2}\right)}\right\}=\frac{\partial \bar{\psi}}{\partial R} \frac{\partial}{\partial \sigma}\left(\frac{D^{2} \bar{\psi}}{R^{2}\left(1-\sigma^{2}\right)}\right)-\frac{\partial \bar{\psi}}{\partial \sigma} \frac{\partial}{\partial R}\left(\frac{D^{2} \bar{\psi}}{R^{2}\left(1-\sigma^{2}\right)}\right) .
$$

\section{Solutions}

In this section, we apply the inverse methods to obtain the exact solutions of the nonlinear partial differential equations (appearing in Section 3) by considering specific forms of the stream function. 
4.1. Flow where $\psi(r, \theta, t)=r^{n} F(\theta, t)$. We choose

$$
\psi(r, \theta, t)=r^{n} F(\theta, t)
$$

in which the arbitrary function $F$ depends upon $\theta$ and $t$, and $n$ is an integer. Upon making use of (4.1), (3.9) can be written as

$$
\begin{aligned}
\rho\left[\frac{\partial G}{\partial t}\right. & \left.-\left\{n F \frac{\partial G}{\partial \theta}-(n-2) \frac{\partial F}{\partial \theta} G\right\} r^{n-2}\right] \\
& =\left(\mu+\alpha_{1} \frac{\partial}{\partial t}\right) H r^{-2}-\alpha_{1}\left[n F \frac{\partial H}{\partial \theta}-(n-4) \frac{\partial F}{\partial \theta} H\right] r^{n-4}
\end{aligned}
$$

In the above equation,

$$
\begin{gathered}
G(\theta, t)=\left(n^{2}+\frac{\partial^{2}}{\partial \theta^{2}}\right) F(\theta, t), \\
H(\theta, t)=\left((n-2)^{2}+\frac{\partial^{2}}{\partial \theta^{2}}\right) G(\theta, t) .
\end{gathered}
$$

Taking $n=0,(4.2)$, and (4.3) yield

$$
\begin{gathered}
\rho \frac{\partial G}{\partial t}=0 \\
2 \rho \frac{\partial F}{\partial \theta} G+\mu H+\alpha_{1} \frac{\partial H}{\partial t}=0 \\
4 \alpha_{1} H \frac{\partial F}{\partial \theta}=0
\end{gathered}
$$

where

$$
G=\frac{\partial^{2} F}{\partial \theta^{2}}, \quad H=4 G+\frac{\partial^{2} G}{\partial \theta^{2}}
$$

It is worth mentioning that for $\alpha_{1}=0$ and $\partial_{t}(\cdot)=0$, we get Jeffery-Hamel flows [21] and for $\partial_{t}(\cdot)=0$, we recover the analysis of reference [3].

Equation (4.4a) implies that $G \neq G(t)$ which shows that $G$ is steady, and hence from (4.5) $H$ is steady. From (4.4c), we assume $\partial F / \partial \theta \neq 0$ (since $\partial F / \partial \theta=0 \Rightarrow F \neq F(\theta)$ which contradicts the assumption (4.1)) which implies that $H=0$. Using this information in (4.4b), we get

$$
2 \rho \frac{\partial F}{\partial \theta} \frac{\partial^{2} F}{\partial \theta^{2}}=0
$$

The solution of the above equation is

$$
F(\theta, t)=A(t) \theta+B(t)
$$

where $A(t)$ and $B(t)$ are arbitrary functions. 
Muhammad R. Mohyuddin et al. 9

Consequently, the expressions for stream function and velocity components are given through (3.2) and (4.1) as

$$
\psi(r, \theta, t)=A(t) \theta+B(t), \quad u=r^{-1} A(t), \quad v=0 .
$$

For $n=1,(4.2)$ becomes

$$
\rho\left[\frac{\partial G}{\partial t}-\left\{F \frac{\partial G}{\partial \theta}+\frac{\partial F}{\partial \theta} G\right\} r^{-1}\right]=\left(\mu+\alpha_{1} \frac{\partial}{\partial t}\right) H r^{-2}-\alpha_{1}\left[F \frac{\partial H}{\partial \theta}+3 \frac{\partial F}{\partial \theta} H\right] r^{-4},
$$

which gives rise to the following equations:

$$
\begin{gathered}
\frac{\partial G}{\partial t}=0, \\
\frac{\partial}{\partial \theta}(F G)=0, \\
\left(\mu+\alpha_{1} \frac{\partial}{\partial t}\right) H=0, \\
F \frac{\partial H}{\partial \theta}+3 \frac{\partial F}{\partial \theta} H=0,
\end{gathered}
$$

where

$$
G=F+\frac{\partial^{2} F}{\partial \theta^{2}}, \quad H=G+\frac{\partial^{2} G}{\partial \theta^{2}}
$$

Equation (4.10a) indicates that $G$ is steady, and hence through (4.11) $H$ is steady and from $(4.10 \mathrm{c})$, we get

$$
\frac{\partial^{2} G}{\partial \theta^{2}}+G=0
$$

whose solution is

$$
G(\theta, t)=A_{1}(t) \cos \theta+B_{1}(t) \sin \theta
$$

where $A_{1}(t)$ and $B_{1}(t)$ are arbitrary functions of $t$. Substitution of (4.13) into (4.10b) yields

$$
F(\theta, t)=C(t)\left[A_{1}(t) \cos \theta+B_{1}(t) \sin \theta\right]^{-1},
$$

where $C(t)$ is a function of integration. The stream function and velocity components are, respectively, given by

$$
\begin{gathered}
\psi(r, \theta, t)=r C(t)\left[A_{1}(t) \cos \theta+B_{1}(t) \sin \theta\right]^{-1}, \\
u=C(t)\left[A_{1}(t) \sin \theta-B_{1}(t) \cos \theta\right]\left[A_{1}(t) \cos \theta+B_{1}(t) \sin \theta\right]^{-2}, \\
v=-C(t)\left[A_{1}(t) \cos \theta+B_{1}(t) \sin \theta\right]^{-1} .
\end{gathered}
$$


For $n=2$, we have from (4.2) that

$$
\rho\left[\frac{\partial G}{\partial t}-2 F \frac{\partial G}{\partial \theta}\right]=\left(\mu+\alpha_{1} \frac{\partial}{\partial t}\right) H r^{-2}-\alpha_{1}\left[2 F \frac{\partial H}{\partial \theta}+2 \frac{\partial F}{\partial \theta} H\right] r^{-2},
$$

which yields

$$
\begin{gathered}
\frac{\partial G}{\partial t}-2 F \frac{\partial G}{\partial \theta}=0 \\
\left(\mu+\alpha_{1} \frac{\partial}{\partial t}\right) H-2 \alpha_{1} \frac{\partial}{\partial \theta}(F H)=0
\end{gathered}
$$

where

$$
G=4 F+\frac{\partial^{2} F}{\partial \theta^{2}}, \quad H=\frac{\partial^{2} G}{\partial \theta^{2}} .
$$

For viscous case $\left(\alpha_{1}=0\right)$, we get

$$
\begin{gathered}
\left(\frac{\partial}{\partial t}-2 F \frac{\partial}{\partial \theta}\right)\left(4 F+\frac{\partial^{2} F}{\partial \theta^{2}}\right)=0, \\
\frac{\partial^{2}}{\partial \theta^{2}}\left(4 F+\frac{\partial^{2} F}{\partial \theta^{2}}\right)=0 .
\end{gathered}
$$

In order to solve (4.19), we introduce the travelling wave solution of the form

$$
F(\theta, t)=\frac{a}{2}+Q(s), \quad s=\theta+a t,
$$

which (for $Q \neq 0$ ) readily gives

$$
\frac{d^{3} Q}{d s^{3}}+4 \frac{d Q}{d s}=0
$$

Solving (4.21) and then inserting in (4.20), we obtain

$$
F(\theta, t)=a_{4}+a_{2} \cos 2(\theta+a t)+a_{3} \sin 2(\theta+a t),
$$

where $a, a_{2}, a_{3}$, and $a_{4}$ are arbitrary constants. The stream function and velocity components are

$$
\begin{gathered}
\psi(r, \theta, t)=r^{2}\left[a_{4}+a_{2} \cos 2(\theta+a t)+a_{3} \sin 2(\theta+a t)\right], \\
u=2 r\left[-a_{2} \sin 2(\theta+a t)+a_{3} \cos 2(\theta+a t)\right], \\
v=-2 r\left[a_{4}+a_{2} \cos 2(\theta+a t)+a_{3} \sin 2(\theta+a t)\right] .
\end{gathered}
$$

For $\alpha_{1} \neq 0,(4.17)$ gives

$$
\begin{gathered}
4 \frac{\partial F}{\partial t}+\frac{\partial^{3} F}{\partial t \partial \theta^{2}}-8 F \frac{\partial F}{\partial \theta}-2 F \frac{\partial^{3} F}{\partial \theta^{3}}=0 \\
\left(\mu+\alpha_{1} \frac{\partial}{\partial t}\right)\left(4 \frac{\partial F}{\partial \theta}+\frac{\partial^{3} F}{\partial \theta^{3}}\right)-2 \alpha_{1}\left(4 F \frac{\partial^{2} F}{\partial \theta^{2}}+\frac{\partial^{4} F}{\partial \theta^{4}}\right)=a_{5}(t)
\end{gathered}
$$


where $a_{5}(t)$ is an arbitrary function. The possible solution of (4.25) for $a_{5}(t)=0$ is given by

$$
\begin{gathered}
\psi(r, \theta, t)=r^{2}\left[\theta_{0} \cos 2 \theta+\theta_{1} \sin 2 \theta\right] e^{\lambda_{2} t}, \\
u=2 r\left[-\theta_{0} \sin 2 \theta+\theta_{1} \cos 2 \theta\right] e^{\lambda_{2} t}, \\
v=-2 r\left[\theta_{0} \cos 2 \theta+\theta_{1} \sin 2 \theta\right] e^{\lambda_{2} t},
\end{gathered}
$$

where $\lambda_{2}, \theta_{0}$, and $\theta_{1}$ are arbitrary constants.

For other values of $n,(4.2)$ requires

$$
\begin{gathered}
\frac{\partial G}{\partial t}=0, \quad\left(\mu+\alpha_{1} \frac{\partial}{\partial t}\right) H=0, \\
n F \frac{\partial G}{\partial \theta}-(n-2) \frac{\partial F}{\partial \theta} G=0, \\
n F \frac{\partial H}{\partial \theta}-(n-4) \frac{\partial F}{\partial \theta} H=0,
\end{gathered}
$$

where $G$ and $H$ are described in (4.3). From (4.27a), we get $G \neq G(t)$, and hence from (4.3b) $H \neq H(t)$ and we obtain that $H=0$. Equation (4.27c) is solved to give the following solution:

$$
G(\theta, t)=C_{1}(t) F^{(n-2) / 2}, \quad n \neq 0,
$$

in which $C_{1}(t)$ is a function of integration.

Equation (4.28) together with (4.3) forms a nonlinear partial differential equation for the determination of $F$ (except when $n=2$ ), which is given as

$$
\frac{\partial^{2} F}{\partial \theta^{2}}+n^{2} F=C_{1}(t) F^{(n-2) / 2} .
$$

The solution for $n=1$ and $C_{1}(t)=0$ is given by

$$
\begin{gathered}
\psi=r\left(A_{3}(t) \cos \theta+B_{3}(t) \sin \theta\right), \\
u=-A_{3}(t) \sin \theta+B_{3}(t) \cos \theta, \\
v=-\left(A_{3}(t) \cos \theta+B_{3}(t) \sin \theta\right) .
\end{gathered}
$$

The solution for $n=2$ and $C_{1}(t) \neq 0$ is as follows:

$$
\begin{gathered}
\psi=r^{2}\left[\frac{C_{1}(t)}{4}+A_{4}(t) \cos 2 \theta+B_{4}(t) \sin 2 \theta\right], \\
u=2 r\left[-A_{4}(t) \sin 2 \theta+B_{4}(t) \cos 2 \theta\right], \\
v=-2 r\left[\frac{C_{1}(t)}{4}+A_{4}(t) \cos 2 \theta+B_{4}(t) \sin 2 \theta\right],
\end{gathered}
$$

in which $A_{i}(t)$ and $B_{i}(t)(i=3,4)$ are arbitrary functions. 
For $\psi=\psi(r, t),(3.9)$ becomes

$$
\left(\mu+\alpha_{1} \frac{\partial}{\partial t}\right) \nabla^{4} \psi-\rho \nabla^{2} \psi_{t}=0
$$

On letting

$$
\psi=\Phi(r) e^{\lambda t}
$$

(4.32) reads as

$$
\frac{1}{r} \frac{\partial}{\partial r}\left[r \frac{\partial}{\partial r}\left\{\frac{1}{r} \frac{\partial}{\partial r}\left(r \frac{\partial \Phi}{\partial r}\right)\right\}\right]-\xi^{2} \frac{1}{r} \frac{\partial}{\partial r}\left(r \frac{\partial \Phi}{\partial r}\right)=0,
$$

which on simplification gives

$$
r^{2} \frac{d^{2} \Phi}{d r^{2}}+r \frac{d \Phi}{d r}-r^{2} \xi^{2} \Phi=\left(A_{4} \ln r+B_{4}\right) r^{2}
$$

where

$$
\xi^{2}=\frac{\rho \lambda}{\mu+\alpha_{1} \lambda} .
$$

For steady case, the solution of (4.32) is

$$
\psi(r)=A_{5} r^{2} \ln r+B_{5} r^{2}+C_{3} \ln r,
$$

where $A_{5}, B_{5}$, and $C_{3}$ are arbitrary constants, and the corresponding velocity components are

$$
u=0, \quad v=-\left(C_{3} r^{-1}+\left(A_{5}+2 B_{5}\right) r+2 A_{5} r \ln r\right)
$$

Here we remark that the solution given in (4.37) is in good agreement to that given by Mohyuddin et al. in [10].

The solution of (4.35) for $A_{4}=B_{4}=0$ is

$$
\psi(r)=\bar{A}_{4} I_{0}(r \xi)+\bar{B}_{4} K_{0}(r \xi)
$$

and the velocity components are

$$
u=0, \quad v=\xi\left[-\bar{A}_{4} I_{1}(r \xi)+\bar{B}_{4} K_{1}(r \xi)\right],
$$

where $I_{n}(x)$ and $K_{n}(x)$ are the modified Bessel functions of the first and second kinds, respectively.

4.2. Flow for $\hat{\psi}(r, z, t)=r^{n} F(z, t)$. Inserting

$$
\widehat{\psi}(r, z, t)=r^{n} F(z, t)
$$


into (3.18), we get

$$
\begin{gathered}
\rho\left[\begin{array}{c}
n(n-2) r^{n-3} \frac{\partial F}{\partial t}+r^{n-1} \frac{\partial^{3} F}{\partial z^{2} \partial t}-4 n(n-2) r^{2 n-5} F \frac{\partial F}{\partial z} \\
-\left\{n F \frac{\partial^{3} F}{\partial z^{3}}-(n-2) \frac{\partial F}{\partial z} \frac{\partial^{2} F}{\partial z^{2}}\right\} r^{2 n-3}
\end{array}\right] \\
=\left(\mu+\alpha_{1} \frac{\partial}{\partial t}\right)\left[\begin{array}{c}
n(n-2)^{2}(n-4) r^{n-5} F+2 n(n-2) r^{n-3} \frac{\partial^{2} F}{\partial z^{2}} \\
+r^{n-1} \frac{\partial^{4} F}{\partial z^{4}}
\end{array}\right] \\
-\alpha_{1}\left[\begin{array}{c}
6 n(n-2)^{2}(n-4) r^{2 n-7} F \frac{\partial F}{\partial z} \\
+2 n(n-2)\left\{n F \frac{\partial^{3} F}{\partial z^{3}}-(n-4) \frac{\partial F}{\partial z} \frac{\partial^{2} F}{\partial z^{2}}\right\} r^{2 n-5}
\end{array}\right] \\
+\left\{\begin{array}{c}
\left.n F \frac{\partial^{5} F}{\partial z^{5}}-(n-2) \frac{\partial F}{\partial z} \frac{\partial^{4} F}{\partial z^{4}}\right\} r^{2 n-3}
\end{array}\right] \\
-2\left(\alpha_{1}+\alpha_{2}\right)\left[\begin{array}{c}
3 n(n-2)^{2}(n-4) r^{2 n-7} F \frac{\partial F}{\partial z}+2(n-2)(3 n-2) r^{2 n-5} \\
\frac{\partial F}{\partial z} \frac{\partial^{2} F}{\partial z^{2}}+\left(\frac{\partial F}{\partial z} \frac{\partial^{4} F}{\partial z^{4}}+2 \frac{\partial^{2} F}{\partial z^{2}} \frac{\partial^{3} F}{\partial z^{3}}\right) r^{2 n-3}
\end{array}\right] .
\end{gathered}
$$

For $n=2$, (4.42) reduces to

$$
\left(\mu+\alpha_{1} \frac{\partial}{\partial t}\right) \frac{\partial^{4} F}{\partial z^{4}}+\rho\left(2 F \frac{\partial^{3} F}{\partial z^{3}}+\frac{\partial^{4} F_{t}}{\partial z^{4}}\right)=2 \alpha_{1}\left(F \frac{\partial^{5} F}{\partial z^{5}}\right)+2\left(\alpha_{1}+\alpha_{2}\right)\left(\frac{\partial F}{\partial z} \frac{\partial^{4} F}{\partial z^{4}}+2 \frac{\partial^{2} F}{\partial z^{2}} \frac{\partial^{3} F}{\partial z^{3}}\right)
$$

The first integral of (4.43) is

$$
\mu \frac{\partial^{3} F}{\partial z^{3}}+\rho\left(2 F \frac{\partial^{2} F}{\partial z^{2}}-\left(\frac{\partial F}{\partial z}\right)^{2}-\frac{\partial F_{t}}{\partial z}\right)=2 \alpha_{1}\left(\begin{array}{c}
F \frac{\partial^{4} F}{\partial z^{4}}-\frac{1}{2} \frac{\partial^{3} F_{t}}{\partial z^{3}} \\
+\left(\frac{\partial^{2} F}{\partial z^{2}}\right)^{2}
\end{array}\right)+\alpha_{2}\left(2 F \frac{\partial^{2} F}{\partial z^{2}}+\left(\frac{\partial^{2} F}{\partial z^{2}}\right)^{2}\right)
$$

where we have taken the function of integration in (4.44) equal to zero. In order to solve (4.44), we define

$$
F(z, t)=N+Q(s), \quad s=z+2 N t,
$$


14 Unsteady semi-inverse solutions

and obtain the following equation:

$$
\mu \frac{d^{3} Q}{d s^{3}}+\rho\left(2 Q \frac{d^{2} Q}{d s^{2}}-\left(\frac{d Q}{d s}\right)^{2}\right)=2 \alpha_{1}\left(Q \frac{d^{4} Q}{d s^{4}}+\left(\frac{d^{2} Q}{d s^{2}}\right)^{2}\right)+\alpha_{2}\left(2 \frac{d Q}{d s} \frac{d^{3} Q}{d s^{3}}+\left(\frac{d^{2} Q}{d s^{2}}\right)^{2}\right)
$$

where $N$ is an arbitrary constant.

Letting $\alpha_{1}=\alpha_{2}=0$ in (4.46) and assuming that

$$
Q=A s^{\lambda}
$$

we get the following relation:

$$
\mu \lambda(\lambda-1)(\lambda-2) s^{\lambda-3}+A \rho \lambda(2(\lambda-1)+1) s^{2(\lambda-1)}=0 .
$$

On choosing $\lambda=-1$, we readily obtain $A=2 \nu$ ( $\nu$ is the kinematic coefficient of viscosity). The expressions for the stream function (4.41) and velocity components (3.15) are

$$
\begin{gathered}
\hat{\psi}(r, z, t)=r^{2}\left[N+2 \nu(z+2 N t)^{-1}\right], \\
u=-2 \nu r(z+2 N t)^{-2}, \\
v=-2\left[N+2 \nu(z+2 N t)^{-1}\right] .
\end{gathered}
$$

It is noted that the solutions (4.49)-(4.51) reduce to that of Berker solutions [3] when $N=0$.

For $\alpha_{1} \neq 0$ and $\alpha_{2} \neq 0$, we assume $[9,21]$ that

$$
Q=A_{0}\left(1+C_{0} e^{a s}\right), \quad s=z+2 N t
$$

in (4.46) and get the following solution:

$$
Q(s)=-\frac{\mu a}{2\left(\rho-\alpha_{1} a^{2}\right)}\left(1+C_{0} e^{a s}\right),
$$

where

$$
a=\sqrt{\rho\left(4 \alpha_{1}+3 \alpha_{2}\right)^{-1}}
$$

and $C_{0}$ is a constant. The stream function and velocity components now become

$$
\begin{gathered}
\hat{\psi}(r, z, t)=\left[N-\frac{\mu a}{2\left(\rho-\alpha_{1} a^{2}\right)}\left(1+C_{0} e^{a(z+2 N t)}\right)\right] r^{2}, \\
u=-\frac{\mu r}{2\left(\rho-\alpha_{1} a^{2}\right)} C_{0} e^{a(z+2 N t)}, \\
v=\left[-N+\frac{\mu a}{2\left(\rho-\alpha_{1} a^{2}\right)}\left(1+C_{0} e^{a(z+2 N t)}\right)\right] 2 r .
\end{gathered}
$$


The above expressions reduce to the results of Berker [3] for $N=0$.

For $n=0,(4.42)$ gives

$$
\begin{gathered}
\rho \frac{\partial^{2} F_{t}}{\partial z^{3}}-\left(\mu+\alpha_{1} \frac{\partial}{\partial t}\right) \frac{\partial^{4} F}{\partial z^{4}}=0 \\
\rho \frac{\partial F}{\partial z} \frac{\partial^{2} F}{\partial z^{2}}=\alpha_{1} \frac{\partial F}{\partial z} \frac{\partial^{4} F}{\partial z^{4}}+\left(\alpha_{1}+\alpha_{2}\right)\left(\frac{\partial F}{\partial z} \frac{\partial^{4} F}{\partial z^{4}}+2 \frac{\partial^{2} F}{\partial z^{2}} \frac{\partial^{3} F}{\partial z^{3}}\right) \\
\left(5 \alpha_{1}+4 \alpha_{2}\right) \frac{\partial F}{\partial z} \frac{\partial^{2} F}{\partial z^{2}}=0 .
\end{gathered}
$$

Since $\partial F / \partial z \neq 0,(4.58)$ implies that

$$
F(z, t)=a_{1}(t) z+a_{2}(t)
$$

where $a_{1}(t)$ and $a_{2}(t)$ are arbitrary functions of time and the above expression leads to the following values of the stream function and velocity components:

$$
\widehat{\psi}=F, \quad u=r^{-1} a_{1}(t), \quad v=0 .
$$

Writing

$$
F(z, t)=\Phi(z) e^{\lambda_{1} t}
$$

into (4.56) and then solving the resulting equation, we obtain

$$
F(z, t)=\left(a_{5} e^{\eta z}+a_{6} e^{-\eta z}-\frac{\left(a_{3} z+a_{4}\right)}{\eta^{2}}\right) e^{\lambda_{1} t},
$$

in which $a_{i}(i=3$ to 6$)$ are arbitrary constants of integration and

$$
\eta^{2}=\frac{\rho \lambda_{1}}{\mu+\alpha_{1} \lambda_{1}}
$$

where $\lambda_{1}$ is a constant. The stream function and velocity components are of the following form:

$$
\widehat{\psi}=F, \quad u=r^{-1} \frac{\partial F}{\partial z}, \quad v=0
$$

4.3. Flow where $\bar{\Psi}(R, \sigma, t)=R^{n} F(\sigma, t)$. On specializing the solution of (3.28) to the form

$$
\bar{\Psi}(R, \sigma, t)=R^{n} F(\sigma, t)
$$


we obtain the following equation:

$$
\begin{aligned}
& \left.\rho\left[\begin{array}{c}
R^{n-2} \frac{\partial G_{1}}{\partial t} \\
n F \frac{\partial G_{1}}{\partial \sigma} \\
-(n-4) \frac{\partial F}{\partial \sigma} G_{1}
\end{array}\right\} R^{2 n-5}\right]=\left(\mu+\alpha_{1} \partial_{t}\right) H_{1} R^{n-4}-\alpha_{1}\left(n F \frac{\partial H_{1}}{\partial \sigma}-H_{1} \frac{\partial F}{\partial \sigma}(n-6)\right) R^{2 n-7} \\
& +\frac{2\left(\alpha_{1}+\alpha_{2}\right)}{1-\sigma^{2}}\left(\begin{array}{c}
\left(1-\sigma^{2}\right) \frac{\partial^{2}}{\partial \sigma^{2}}\left(G \frac{\partial F}{\partial \sigma}+n \sigma F G_{1}\right) \\
+(2 n-5)(2 n-6) \\
\times\left(G \frac{\partial F}{\partial \sigma}+n \sigma F G_{1}\right) \\
-\left\{G \frac{\partial G}{\partial \sigma}+\frac{(n-2) \sigma}{1-\sigma^{2}} G^{2}\right\}
\end{array}\right) R^{2 n-7},
\end{aligned}
$$

where

$$
\begin{gathered}
G(\sigma, t)=n(n-1) F+\left(1-\sigma^{2}\right) \frac{\partial^{2} F}{\partial \sigma^{2}}, \quad G_{1}(\sigma, t)=G\left(1-\sigma^{2}\right)^{-1}, \\
H(\sigma, t)=(n-2)(n-3) G+\left(1-\sigma^{2}\right) \frac{\partial^{2} G}{\partial \sigma^{2}}, \quad H_{1}=H\left(1-\sigma^{2}\right)^{-1} .
\end{gathered}
$$

For $n=0$, we obtain

$$
\begin{gathered}
\frac{\partial G_{1}}{\partial t}=0, \quad \frac{\partial F}{\partial \sigma} G_{1}=0, \quad\left(\mu+\alpha_{1} \frac{\partial}{\partial t}\right) H_{1}=0 \\
-3 H_{1} \alpha_{1} \frac{\partial F}{\partial \sigma}=-\frac{\left(\alpha_{1}+\alpha_{2}\right)}{1-\sigma^{2}}\left[\begin{array}{c}
\left(1-\sigma^{2}\right) \frac{\partial^{2}}{\partial \sigma^{2}}\left(G \frac{\partial F}{\partial \sigma}\right)+30 G \frac{\partial F}{\partial \sigma} \\
-\left(G \frac{\partial G}{\partial \sigma}-2 \frac{\sigma}{1-\sigma^{2}} G^{2}\right)
\end{array}\right] .
\end{gathered}
$$

Equations (4.68) $)_{1,3}$ and (4.67) $)_{2,4}$ imply that $G$ and $H$ are not functions of $t$, and hence $(4.68)_{2}$ becomes

$$
\left(1-\sigma^{2}\right) \frac{\partial F}{\partial \sigma} \frac{\partial^{2} F}{\partial \sigma^{2}}=0 .
$$

Since $\partial F / \partial \sigma \neq 0$, the above equation yields

$$
F(\sigma, t)=\bar{C}_{0}(t) \sigma+\bar{C}_{1}(t)
$$

where $\bar{C}_{0}$ and $\bar{C}_{1}$ are arbitrary functions and the stream function and velocity components are

$$
\bar{\Psi}=\bar{C}_{0}(t) \sigma+\bar{C}_{1}(t), \quad u=\frac{\bar{C}_{0}(t)}{R^{2}}, \quad v=0 .
$$


Now (4.66) for $n=1$ leads to the following:

$$
\begin{gathered}
\frac{\partial G_{1}}{\partial t}=0, \quad-\rho\left\{F \frac{\partial G_{1}}{\partial \sigma}+3 \frac{\partial F}{\partial \sigma} G_{1}\right\}=\left(\mu+\alpha_{1} \frac{\partial}{\partial t}\right) H_{1} \\
-\alpha_{1}\left(F \frac{\partial H_{1}}{\partial \sigma}+5 H_{1} \frac{\partial F}{\partial \sigma}\right)+\frac{2\left(\alpha_{1}+\alpha_{2}\right)}{1-\sigma^{2}} \\
\times\left[\left(1-\sigma^{2}\right) \frac{\partial^{2}}{\partial \sigma^{2}}\left(G \frac{\partial F}{\partial \sigma}+\sigma F G_{1}\right) 12\left(G \frac{\partial F}{\partial \sigma}+\sigma F G_{1}\right)-\left(G \frac{\partial G}{\partial \sigma}+\frac{\sigma}{1-\sigma^{2}} G^{2}\right)\right]=0
\end{gathered}
$$

where

$$
\begin{gathered}
G(\sigma, t)=\left(1-\sigma^{2}\right) \frac{\partial^{2} F}{\partial \sigma^{2}}, \quad G_{1}=\left(1-\sigma^{2}\right)^{-1} G, \\
H(\sigma, t)=2 G+\left(1-\sigma^{2}\right) \frac{\partial^{2} G}{\partial \sigma^{2}}, \quad H_{1}=\left(1-\sigma^{2}\right)^{-1} H,
\end{gathered}
$$

and (4.3) implies that $G_{1}$ is steady, and hence $H_{1}$ is steady. Using (4.73) in (4.3), we obtain

$$
\frac{\partial^{3}}{\partial \sigma^{3}}\left[2 \mu\left(1-\sigma^{2}\right) \frac{\partial F}{\partial \sigma}+4 \mu \sigma F+\rho F^{2}\right]=0 .
$$

Various steady cases of the above equation have been discussed by Squire [21]. Integrating (4.74) and employing a similar procedure as used by Landau and Lifshitz [9] (after setting the functions of integrations equal to zero), we obtain a particular solution of the form

$$
F(\sigma, t)=2 \nu\left(1-\sigma^{2}\right)(\sigma-a)^{-1}
$$

where $a$ is a constant.

Substitution of (4.75) into $(4.3)_{3}$ yields

$$
\begin{aligned}
96 v^{2}\left[\frac{1}{(\sigma-a)^{2}}-\frac{2 \sigma}{(\sigma-a)^{3}}-\frac{1-\sigma^{2}}{(\sigma-a)^{4}}\right] \\
\quad \times\left[\alpha_{1}\left\{\frac{11 \sigma^{2}-1}{\sigma-a}+\frac{21 \sigma\left(1-\sigma^{2}\right)}{(\sigma-a)^{2}}+\frac{10\left(1-\sigma^{2}\right)^{2}}{(\sigma-a)^{3}}\right\}\left(\alpha_{1}+\alpha_{2}\right)\right. \\
\left.\quad \times\left\{2 \sigma+\frac{1+\sigma^{2}}{\sigma-a}+\frac{7 \sigma\left(1-\sigma^{2}\right)}{(\sigma-a)^{2}}+\frac{4 \sigma\left(1-\sigma^{2}\right)^{2}}{(\sigma-a)^{3}}\right\}\right]=0 .
\end{aligned}
$$

We note that the solution (4.75) cannot be obtained for all values of $a$. Siddiqui and Kaloni [19] obtained the solution for the steady case when $a=-1,0,1$. For the sake of completeness, we include it here briefly for the convenience of the reader. Setting $a= \pm 1$, (4.76) is identically satisfied and (4.75) gives

$$
F_{1,2}=\mp 2 \nu(1 \pm \sigma) \quad \text { for } a= \pm 1 \text {. }
$$


For $a=0$ and $7 \alpha_{1}+2 \alpha_{2}=0,(4.75)$ becomes

$$
F_{3}=\frac{2 v}{\sigma}\left(1-\sigma^{2}\right)
$$

The stream function and velocity fields for the above three cases are

$$
\begin{gathered}
\bar{\Psi}=R F_{1}(\sigma, t), \quad u=-\frac{2 v}{R}, \quad v=-\frac{2 v}{R} \frac{(1+\sigma)}{\sqrt{1-\sigma^{2}}}, \\
\bar{\Psi}=R F_{2}(\sigma, t), \quad u=-\frac{2 v}{R}, \quad v=\frac{2 v}{R} \frac{(1-\sigma)}{\sqrt{1-\sigma^{2}}}, \\
\bar{\Psi}=R F_{3}(\sigma, t), \quad u=-\frac{2 v}{R} \frac{1+\sigma^{2}}{\sigma^{2}}, \quad v=\frac{2 v}{R} \frac{\sqrt{1-\sigma^{2}}}{\sigma} .
\end{gathered}
$$

For $n=2$, (4.66) yields the following form:

$$
\begin{gathered}
\frac{\partial G_{1}}{\partial t}=0, \quad \frac{\partial}{\partial \sigma}\left(G_{1} F\right)=0, \quad\left(\mu+\alpha_{1} \partial_{t}\right) H_{1}=0 \\
-\alpha_{1}\left(F \frac{\partial H_{1}}{\partial \sigma}+2 H_{1} \frac{\partial F}{\partial \sigma}\right)+\frac{\left(\alpha_{1}+\alpha_{2}\right)}{1-\sigma^{2}} \\
\times\left[\left(1-\sigma^{2}\right)+\frac{\partial^{2}}{\partial \sigma^{2}}\left(G \frac{\partial F}{\partial \sigma}+2 \sigma F G_{1}\right)+2\left(G \frac{\partial F}{\partial \sigma}+2 \sigma F G_{1}\right)-G \frac{\partial G}{\partial \sigma}\right]=0
\end{gathered}
$$

in which

$$
\begin{gathered}
G=2 F+\left(1-\sigma^{2}\right) \frac{\partial^{2} F}{\partial \sigma^{2}}, \quad G_{1}=\left(1-\sigma^{2}\right)^{-1} G \\
H=\left(1-\sigma^{2}\right) \frac{\partial^{2} G}{\partial \sigma^{2}}, \quad H_{1}=\left(1-\sigma^{2}\right)^{-1} H .
\end{gathered}
$$

Equation $(4.80)_{2}$ can also be written as

$$
\left(1-\sigma^{2}\right)^{-1} F\left[2 F+\left(1-\sigma^{2}\right) \frac{\partial^{2} F}{\partial \sigma^{2}}\right]=\bar{C}(t) .
$$

The solution of the above equation is

$$
F(\sigma, t)=\left(\sigma^{2}-1\right) \widetilde{C}_{1}(t)+\frac{1}{4} \widetilde{C}_{2}(t)\left[-2 \sigma+\left(1-\sigma^{2}\right)\{\ln (\sigma-1)-\ln (\sigma+1)\}\right]
$$

and the expressions for the stream function and velocity components are

$$
\begin{gathered}
\bar{\Psi}=R^{2} F(\sigma, t), \quad v=2\left(1-\sigma^{2}\right)^{-1 / 2} F(\sigma, t), \\
u=\frac{\sigma}{2}\left[4 \widetilde{C}_{1}(t)+\widetilde{C}_{2}(t)\left\{\ln (\sigma+1)-\ln (\sigma-1)-\frac{2}{\sigma}\right\}\right] .
\end{gathered}
$$


For $n=3$, (4.66) gives rise to

$$
\begin{gathered}
\frac{\partial G_{1}}{\partial t}-\left(3 F \frac{\partial G_{1}}{\partial \sigma}+\frac{\partial F}{\partial \sigma} G_{1}\right)=0 \\
\left(\mu+\alpha_{1} \partial_{t}\right) H_{1}-\alpha_{1}\left(3 \frac{\partial H_{1}}{\partial \sigma} F+3 H_{1} \frac{\partial F}{\partial \sigma}\right) \\
=-2\left(\alpha_{1}+\alpha_{2}\right)\left[\frac{\partial^{2}}{\partial \sigma^{2}}\left(G \frac{\partial F}{\partial \sigma}+3 \sigma F G_{1}\right)-\left(G_{1} \frac{\partial G}{\partial \sigma}+\sigma G_{1}^{2}\right)\right],
\end{gathered}
$$

where

$$
\begin{gathered}
G=6 F+\left(1-\sigma^{2}\right) \frac{\partial^{2} F}{\partial \sigma^{2}}, \quad G_{1}=\left(1-\sigma^{2}\right)^{-1} G, \\
H=\left(1-\sigma^{2}\right) \frac{\partial^{2} G}{\partial \sigma^{2}}, \quad H_{1}=\left(1-\sigma^{2}\right)^{-1} H .
\end{gathered}
$$

For the steady and viscous cases, (4.86) gives $\mu H_{1}=0$ which on using $(4.87)_{3}$ becomes

$$
\left(1-\sigma^{2}\right) \frac{\partial^{2} F}{\partial \sigma^{2}}+6 F=k_{1} \sigma+k_{2}
$$

in which $k_{1}$ and $k_{2}$ are integration constants. We see that the solution $F$ obtained from (4.88) will only be satisfied by (4.85) when $k_{1}=k_{2}=0$. The solution of (4.88) is given as

$$
\bar{\Psi}=R^{3} F(\sigma), \quad u=R F_{1}(\sigma), \quad v=3 R\left(1-\sigma^{2}\right)^{-1 / 2} F_{1}(\sigma) .
$$

In (4.89),

$$
\begin{aligned}
& F(\sigma)=\sigma\left(\sigma^{2}-1\right) \widetilde{C}_{3}-\frac{1}{4} \widetilde{C}_{4}\left[\begin{array}{c}
-4+6 \sigma^{2} \\
+3 \sigma\left(\sigma^{2}-1\right)(1-\ln (1+\sigma))+\ln (\sigma-1)
\end{array}\right], \\
& F_{1}(\sigma)=\left(3 \sigma^{2}-1\right) \widetilde{C}_{3}-\frac{9}{2} \widetilde{C}_{4} \sigma+\frac{3}{4}\left(3 \sigma^{2}-1\right) \widetilde{C}_{4}\{\ln (1+\sigma)-\ln (\sigma-1)\} .
\end{aligned}
$$

For $k_{1} \neq 0$ and $k_{2} \neq 0$, we have the following solutions:

$$
\bar{\Psi}=R^{3} F(\sigma), \quad u=R \frac{d F}{d \sigma}, \quad v=3 R\left(1-\sigma^{2}\right)^{-1 / 2} F(\sigma),
$$


in which

$$
F(\sigma)=\frac{1}{24}\left[\begin{array}{c}
c 6 k_{2} \sigma^{2}+4\left\{k_{1} \sigma^{3}+6 \sigma\left(\sigma^{2}-1\right) \widetilde{C}_{5}+3 \widetilde{C}_{6}\left(2-3 \sigma^{2}\right)\right\} \\
+3 \sigma\left(\sigma^{2}-1\right)\left(k_{2}-6 \widetilde{C}_{6}\right)\{\ln (\sigma-1)-\ln (\sigma+1)\}
\end{array}\right],
$$

where $\tilde{C}_{i}(i=3$ to 6$)$ are constants.

For $n=4$, (4.66) reduces to

$$
\begin{aligned}
\rho\left[R^{2} \frac{\partial G_{1}}{\partial t}-4 F \frac{\partial G_{1}}{\partial \sigma} R^{3}\right]= & \left(\mu+\alpha_{1} \partial_{t}\right) H_{1}-\alpha_{1}\left[4 F \frac{\partial H_{1}}{\partial \sigma}+2 H_{1} \frac{\partial F}{\partial \sigma}\right] R \\
& +\frac{2\left(\alpha_{1}+\alpha_{2}\right)}{\left(1-\sigma^{2}\right)}\left[\left(1-\sigma^{2}\right) \frac{\partial^{2}}{\partial \sigma^{2}}\left(G \frac{\partial F}{\partial \sigma}+4 \sigma F G_{1}\right)\right. \\
& \left.+6\left(G \frac{\partial F}{\partial \sigma}+4 \sigma F G_{1}\right)-\left(G \frac{\partial G}{\partial \sigma}+2 \sigma\left(1-\sigma^{2}\right)^{-1} G^{2}\right)\right] R
\end{aligned}
$$

where

$$
\begin{array}{ll}
G=\left(1-\sigma^{2}\right) \frac{\partial^{2} F}{\partial \sigma^{2}}+12 F, & G_{1}=\left(1-\sigma^{2}\right)^{-1} G, \\
H=\left(1-\sigma^{2}\right) \frac{\partial^{2} G}{\partial \sigma^{2}}+2 G, & H_{1}=\left(1-\sigma^{2}\right)^{-1} H .
\end{array}
$$

The following equations are obtained from (4.93):

$$
\begin{gathered}
\frac{\partial G_{1}}{\partial t}=0, \quad F \frac{\partial G_{1}}{\partial \sigma}=0, \quad\left(\mu+\alpha_{1} \partial_{t}\right) H_{1}=0 \\
\alpha_{1}\left(2 F \frac{\partial H_{1}}{\partial \sigma}+2 H_{1} \frac{\partial F}{\partial \sigma}\right)=\left(\alpha_{1}+\alpha_{2}\right)\left[\begin{array}{c}
\left(1-\sigma^{2}\right) \frac{\partial^{2}}{\partial \sigma^{2}}\left(G \frac{\partial F}{\partial \sigma}+4 \sigma F G_{1}\right) \\
+6\left(G \frac{\partial F}{\partial \sigma}+4 \sigma F G_{1}\right) \\
-\left(G \frac{\partial G}{\partial \sigma}+2 \sigma\left(1-\sigma^{2}\right)^{-1} G^{2}\right)
\end{array}\right] .
\end{gathered}
$$

The first and third equations in (4.95) imply that $G_{1}$ is steady, and hence $H_{1}$ is steady. Since $F \neq 0,(4.95)_{2}$ gives $\partial G_{1} / \partial \sigma=0$, which on using (4.94) have the following solution:

$$
G=A_{6}\left(1-\sigma^{2}\right)
$$

On substituting (4.97) in (4.95) 1 , we can write

$$
\left(1-\sigma^{2}\right) \frac{\partial^{2} F}{\partial \sigma^{2}}+12 F=A_{6}\left(1-\sigma^{2}\right)
$$


The solution of (4.98) for steady case is given by Berker [3]. In order to avoid repetition, we directly give the solution with the stream function and velocity components as

$$
\begin{gathered}
F=k_{3} \sigma^{2}\left(1-\sigma^{2}\right), \\
\bar{\Psi}=k_{3} \sigma\left(1-2 \sigma^{2}\right) R^{2}, \\
u=2 k_{3} \sigma\left(1-2 \sigma^{2}\right) R^{2}, \quad v=4 k_{3} \sigma^{2} \sqrt{1-\sigma^{2}} R^{2},
\end{gathered}
$$

where $k_{3}$ is a constant.

\section{Concluding remarks}

In this paper, the governing time-dependent equations for plane polar, axisymmetric cylindrical, and spherical coordinates are constructed. Moreover, the analytical solutions for eleven nonlinear equations involving three-coordinate systems are given. The solutions obtained are found to be in good agreement to that of the previous steady solutions for viscous and second-grade fluids.

\section{Acknowledgments}

The authors gratefully acknowledge the support from URF Scheme of Quaid-i-Azam University, Islamabad, Pakistan. Muhammad R. Mohyuddin is thankful to Dr. Arshad M. Khan (Director of Global Change Impact Studies Centre (GCISC)) and Dr. Shoaib Raza for their nice cooperation and encouragement in order to continue his research.

\section{References}

[1] R. Bandelli, K. R. Rajagopal, and G. P. Galdi, On some unsteady motions of fluids of second grade, Archives of Mechanics 47 (1995), no. 4, 661-676.

[2] A. M. Benharbit and A. M. Siddiqui, Certain solutions of the equations of the planar motion of a second grade fluid for steady and unsteady cases, Acta Mechanica 94 (1992), no. 1-2, 85-96.

[3] R. Berker, Intégration des équations du mouvement d'un fluide visqueux incompressible, Handbuch der Physik, Bd. VIII/2, Springer, Berlin, 1963, pp. 1-384.

[4] J. E. Dunn and K. R. Rajagopal, Fluids of differential type: critical review and thermodynamic analysis, International Journal of Engineering Science 33 (1995), no. 5, 689-729.

[5] R. L. Fosdick and K. R. Rajagopal, Anomalous features in the model of "second order fluids", Archive for Rational Mechanics and Analysis 70 (1979), no. 2, 145-152.

[6] T. Hayat, M. R. Mohyuddin, and S. Asghar, Some inverse solutions for unsteanian fluid, Tamsui Oxford Journal of Mathematical Sciences 21 (2005), no. 1, 1-20.

[7] P. N. Kaloni and K. Huschilt, Semi-inverse solutions of a non-Newtonian fluid, International Journal of Non-Linear Mechanics 19 (1984), no. 4, 373-381.

[8] F. Labropulu, Exact solutions of non-Newtonian fluid flows with prescribed vorticity, Acta Mechanica 141 (2000), no. 1-2, 11-20.

[9] L. D. Landau and E. M. Lifshitz, Fluid Mechanics, Course of Theoretical Physics, vol. 6, Pergamon Press, London, 1959.

[10] M. R. Mohyuddin, T. Hayat, F. M. Mahomed, S. Asghar, and A. M. Siddiqui, On solutions of some non-linear differential equations arising in Newtonian and non-Newtonian fluids, Nonlinear Dynamics 35 (2004), no. 3, 229-248. 
[11] K. R. Rajagopal, On the decay of vortices in a second grade fluid, Meccanica 15 (1980), no. 3, $185-186$.

[12] _ A note on unsteady unidirectional flows of a non-Newtonian fluid, International Journal of Non-Linear Mechanics 17 (1982), no. 5-6, 369-373.

[13] _ Boundedness and uniqueness of fluids of the differential type, Acta Ciencia Indica. Mathematics 8 (1982), no. 1-4, 28-38.

[14] - On boundary conditions for fluids of the differential type, Navier-Stokes Equations and Related Nonlinear Problems (Funchal, 1994) (A. Sequeira, ed.), Plenum Press, New York, 1995, pp. 273-278.

[15] K. R. Rajagopal and A. S. Gupta, On a class of exact solutions to the equations of motion of a second grade fluid, International Journal of Engineering Science 19 (1981), no. 7, 1009-1014.

[16] _ An exact solution for the flow of a non-Newtonian fluid past an infinite porous plate, Meccanica 19 (1984), no. 2, 158-160.

[17] K. R. Rajagopal and P. N. Kaloni, Some remarks on boundary conditions for flows of fluids of the differential type, Continuum Mechanics and Its Applications (G. A. C. Graham and S. K. Malik, eds.), Hemisphere, District of Columbia, 1999, pp. 935-941.

[18] R. S. Rivlin and J. L. Ericksen, Stress-deformation relations for isotropic materials, Journal of Rational and Mechanical Analysis 4 (1955), 323-425.

[19] A. M. Siddiqui and P. N. Kaloni, Certain inverse solutions of a non-Newtonian fluid, International Journal of Non-Linear Mechanics 21 (1986), no. 6, 459-473.

[20] A. M. Siddiqui, M. R. Mohyuddin, T. Hayat, and S. Asghar, Some more inverse solutions for steady flows of a second-grade fluid, Archives of Mechanics 55 (2003), no. 4, 373-387.

[21] H. B. Squire, The round laminar jet, Quarterly Journal of Mechanics \& Applied Mathematics 69 (1979), 335-380.

Muhammad R. Mohyuddin: Department of Mathematics, Quaid-i-Azam University, Islamabad-44000, Pakistan

Current address: System and Decision Sciences, Global Change Impact Studies Centre, 61/A, 1st Floor, Saudi-Pak Tower, Constitutive Avenue, Islamabad, Pakistan

E-mail address: m_raheel@yahoo.com

S. Asghar: COMSATS Institute of Information Technology, Plot 30, Sector H-8, Islamabad, Pakistan

E-mail address: s_asgharpk@yahoo.com

T. Hayat: Department of Mathematics, Quaid-i-Azam University, Islamabad-44000, Pakistan E-mail address: t_pensy@hotmail.com

A. M. Siddiqui: Department of Mathematics, Pennsylvania State University, York Campus, York, PA 17403, USA

E-mail address: ams5@psu.edu 


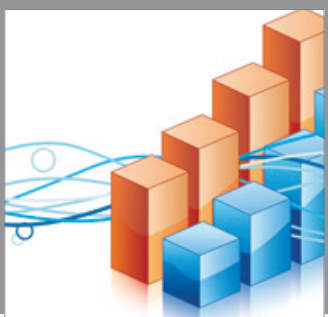

Advances in

Operations Research

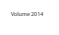

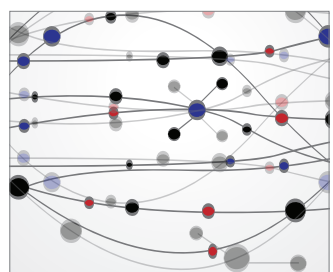

\section{The Scientific} World Journal
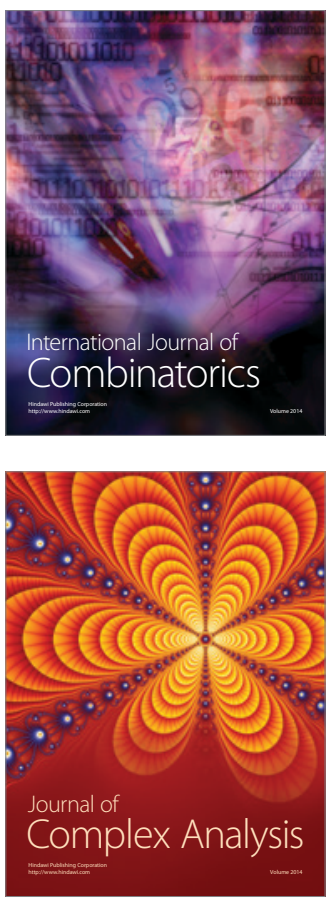

International Journal of

Mathematics and

Mathematical

Sciences
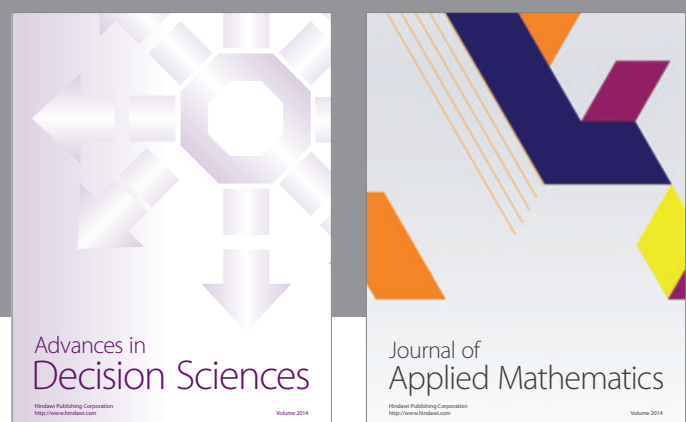

Journal of

Applied Mathematics
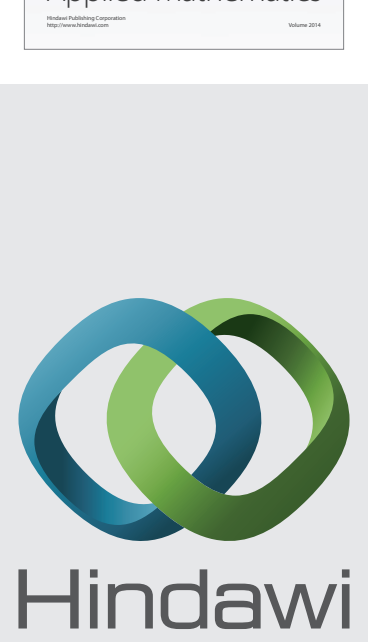

Submit your manuscripts at http://www.hindawi.com
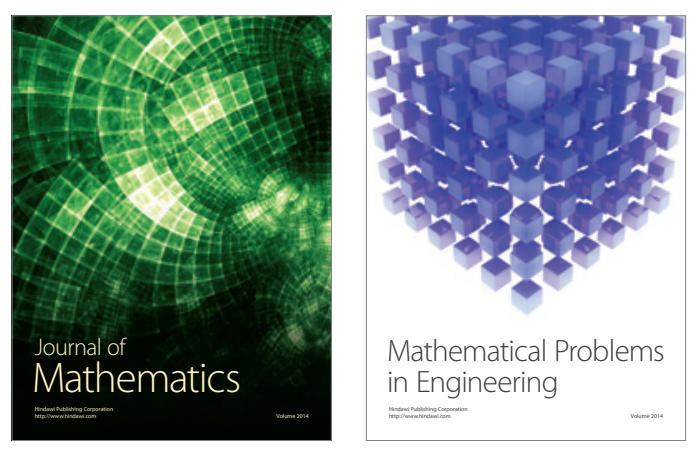

Mathematical Problems in Engineering
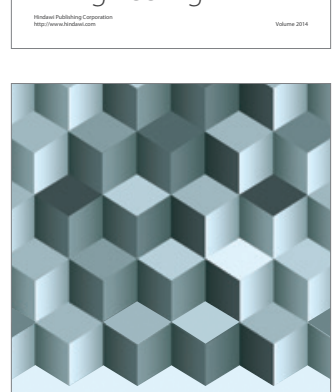

Journal of

Function Spaces
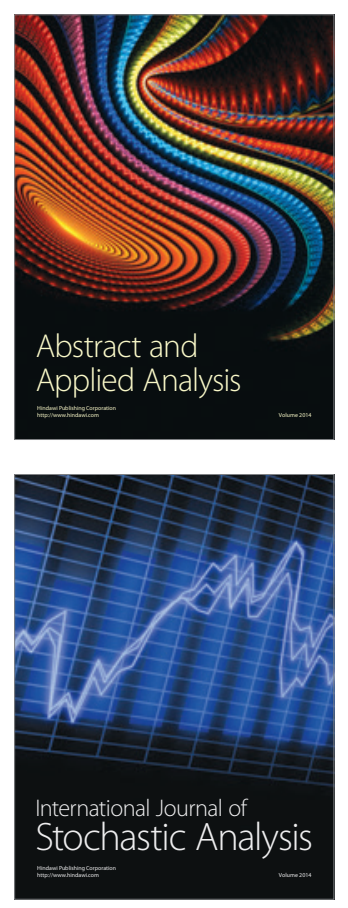

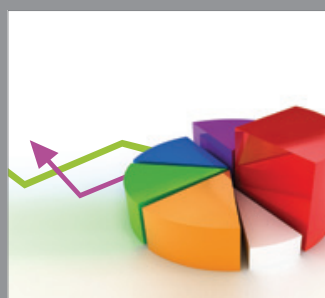

ournal of

Probability and Statistics

Promensencen
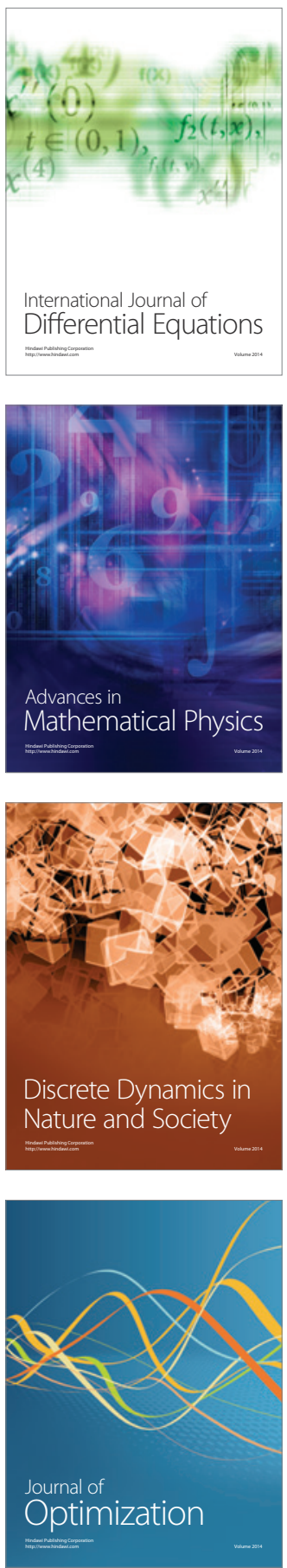